\title{
Cloning of the Recombinant Cytochrome P450 Cyp141 Protein of Mycobacterium tuberculosis as a Diagnostic Target and Vaccine Candidate
}

\author{
Mohammad Rabiee-Faradonbeh ${ }^{1,2}$; Davood Darban-Sarokhalil ${ }^{3}$; Mohammad Mehdi \\ Feizabadi ${ }^{4}$;Amirhooshang Alvandi ${ }^{5}$; Hasan Momtaz ${ }^{2}$;Neda Soleimani ${ }^{6}$;Abolfazl Gholipour ${ }^{1, *}$ \\ ${ }_{2}^{1}$ Department of Microbiology and Immunology, Faculty of Medicine, Cellular and Molecular Research Center, Shahrekord University of Medical Sciences, Shahrekord, IR Iran \\ ${ }_{3}^{2}$ Department of Microbiology, Shahrekord Branch, Islamic Azad University, Shahrekord,IR Iran \\ ${ }^{3}$ Department of Microbiology and Immunology, Faculty of Medicine, Alborz University of Medical Sciences, Karaj, IR Iran \\ ${ }_{5}^{4}$ Department of Microbiology, Faculty of Medicine, Tehran University of Medical Sciences, Tehran, IR Iran \\ 5 Department of Microbiology, Faculty of Medicine, Kermanshah University of Medical Sciences, Kermanshah, IR Iran \\ 6 Department of Pathology, Al-Zahra Hospital, Isfahan University of Medical Sciences, Isfahan, IR Iran \\ ${ }^{*}$ Corresponding Author: Abolfazl Gholipour, Department of Microbiology and Immunology, Cellular and Molecular Research Center, Faculty of Medicine, Shahrekord University of \\ Medical Sciences, Rahmatiyeh, Shahrekord, IR Iran. Tel:+98-3813346732, E-mail: gholipour_abolfazl@yahoo.com
}

Received: February 9, 2014; Revised: April 8, 2014; Accepted: April 23, 2014

\begin{abstract}
Background: Tuberculosis has been announced as a global emergency by World Health Organization and the second infectious agent of mortality worldwide. The general policy in the development of new vaccines is to develop some vaccines with higher efficiency not only for infants but also for adults compared with the Bacillus Calmette-Guerin vaccine. Recently, cytochrome P450 cyp141 has been introduced as a new target for detecting Mycobacterium tuberculosis from clinical samples.

Objectives: The aim of this study was to clone this gene in order to pave the way for more evaluation.

Materials and Methods: M. tuberculosis H37Rv DNA was extracted by a standard phenol-chlorophorm protocol. After designing the specific primers, P450 cyp141 gene was replicated by PCR. The purified PCR products were then subcloned into the pTZ57R/T plasmid vector. After extraction, enzyme digestion, and recombinant pTZ57R/T-cyp141 plasmid vector sequencing, the aforementioned products were cloned into a pET-26b plasmid vector. Then, the recombinant pET26b-cyp141 plasmid molecules were transformed to Escherichia coli strain BL21 (DE3) using the transformation method. Next, the recombinant pET26b-cyp141 plasmids were purified and evaluated by the enzyme digestion analysis.

Results: The cloning of P450 cyp141 gene was confirmed by the enzyme digestion and sequencing of the recombinant pTZ57R/T-cyp141 and pET26b-cyp141 plasmid vectors.

Conclusions: The results of this study demonstrated that the P450 cyp141 gene was successfully cloned into a pET26b plasmid vector as an expression vector. In this paper, for the first time in Iran, this gene was cloned for more purposes, including the expression and purification of the recombinant cytochrome P450 cyp141 protein.
\end{abstract}

Keywords:Cloning; Cytochrome; Mycobacterium; Tuberculosis; Diagnostic

\section{Background}

Despite more than half a century of worldwide vaccination, Mycobacterium tuberculosis still remains asone of the most common infectious agents in the world (1). Presently, almost one-third of the world's population is thought infected by this pathogen and about 9 million people are infected per year, with nearly 1.4 million deaths (2). During the next 25 years it is anticipated that more than 40 million people may be killed by tuberculosis, unless the control measures are implemented (3). Effective vaccine(s) with capability of protecting against all forms of the disease across the world and the rapid and sensitive diagnostic methods are the most important factors for global control of tuberculosis (4).
Recently a lot of conventional and molecular methods have been introduced for the detection of tuberculosis. Molecular methods, such as DNA probes and nucleic acid amplification tests, are the rapid, sensitive, and specific methods for the detection of $M$. tuberculosis from clinical specimens (3). Different studies have shown the advantages or disadvantages of these methods; however, these procedures are often used for the detection of active tuberculosis, not latent type. Nowadays, serological methods for detection of latent tuberculosis have also been developed, but still a lot of efforts should be made to find the suitable diagnostic target. Moreover, these methods have been debated for correct detection 
of tuberculosis (5). Another strategy for worldwide control of tuberculosis is applying the efficient vaccines. $M$. bovis Bacillus Calmette-Guérin (BCG) is the only effective existent vaccine for control of tuberculosis (6). However, the effect of BCG in adults has been varied from 0 to $80 \%$ and it cannot protect against pulmonary tuberculosis. Moreover since it is a live vaccine, it cannot be used in immunocompromised patients (7). Recently many studies have been done on different targets as vaccine candidate (8). Despite recent studies, tuberculosis remains as an endemic disease in most parts of the world. From the studied targets, there are some regions in M. tuberculosis and $M$. bovis BCG genome sequences called regions of differences, some of which are absent in some or all of $M$. bovis and $M$. bovis BCG strains (9). The main targets of these regions that most studies have been focused on are ESAT- 6 and CFP10. One of the proteins that belong to these regions is cyp141 or Rv3121. The cyp141 gene is one of the conserved genes that interferes in oxidation reduction (10) and has been introduced and used as target for direct detection of tuberculosis by DarbanSarokhalil et al. (11). Mustafa et al. confirmed that this target is absent in all M. bovis and M. bovis BCG strains and they have also been cloned and evaluated for several regions of differences (12). To the best of our knowledge, the recombinant cytochrome $\mathrm{P} 450$ cyp141 protein has not been cloned and evaluated.

\section{Objectives}

The purpose of this study was to clone the target for evaluation in future as a vaccine candidate and possibly the diagnostic target.

\section{Materials and Methods}

\subsection{Evaluation of the Target in Terms of the Immu- nogenicity in Silico}

Prior to performing the laboratory experiments, the target was evaluated in terms of immunogenicity by the ProPred I and II software.

\subsection{Bacterial Strains, Plasmid, Media, and Culture Conditions}

This experimental study was performed from September 2012 to July 2013 in Shahrekord University of Medical Sciences in Iran. M. tuberculosis H37Rv (ATCC 25618) obtained from the American Type Culture Collection (Manassas, Virginia) was cultured on the standard 7H12 broth (Johnston Laboratories, Inc., Towson, Maryland) in 4.0-mL vials. A 50\% egg yolk enrichment solution (Difco Laboratories, Detroit, Michigan) was added at a volume of $0.1 \mathrm{~mL}$ per $7 \mathrm{H} 12$ broth vial before inoculation. The $E$. coli strain JM107 (Fermentas) and plasmid pTZ57R/T (Ins
TA clone PCR Cloning kit, Fermentas) were used for initial molecular cloning and sequencing, respectively. The ampicillin or kanamycin antibiotics (50 mg/L) were added to Luria-Bertani (LB) broth according to the reference recommendation (13). The E. coli strain JM107 and E. coli strain BL21 (DE3) (Novagen Inc., Madison, Wisconsin) were used for cloning and expression, respectively, and the expression vector pET26b (Novagen Inc., Madison, Wisconsin) was used for cloning and also as the T7-based expression vector. All chemicals used in this study were from Merck Company (Germany) and all of the enzymes from Roche (Germany), Fermentas (Lithuania), and Cinagen (Iran) Companies.

\subsection{Primers Design}

The genomic DNA of M. tuberculosis H37Rv and the pET26b plasmid were extracted using the Genomic DNA Purification and GeneJET Plasmid Miniprep kits, respectively, according to the manufacturer's instructions (Fermentas, Lithuania). PCR primers were designed based on the full protein-coding region of the $M$. tuberculosis H37Rv P450 cyp141 gene of the 44-kDa protein by the DNASIS v2.6 Primer Designer Software. The forward primer, cyp141-F, (5' CYP 141-F5'-CGC CAT ATG GCG ACA AGC ACC TCG ATT CC -3') contained an NdeI recognition site and the reverse primer, cyp141-R, (CYP 141-R-CCC AAG CTT GGG CGT CGG CCA GGT AA -3') contained a recognition site for HindIII.

\subsection{Gene Amplification of Cyp141 (Encoding the p450 Protein)}

PCR was performed in a $50 \mu \mathrm{L}$ total volume containing 1 $\mu \mathrm{g}$ of template DNA, $1.5 \mu \mathrm{M}$ of each primer, $1.5 \mathrm{mM} \mathrm{MgCl} 2$, $150 \mu \mathrm{M}$ dNTP Mix, 1x PCR buffer, and 1.5 unit of Taq DNA polymerase (Sigma). The PCR reaction was performed on a Thermocycler TC-512 (Techne, England) and the following conditions were used for amplification: the initial denaturation at $94^{\circ} \mathrm{C}$ for 5 minutes, followed by 30 cycles of denaturation at $94^{\circ} \mathrm{C}$ for 1 minute, annealing at $65^{\circ} \mathrm{C}$ for 40 second, and an extension at $72^{\circ} \mathrm{C}$ for 1 minute. This was then followed by a final extension at $72^{\circ} \mathrm{C}$ for 3 minutes. The PCR product was evaluated by electrophoresis in $1 \%$ agarose gel and visualized by GelRed staining on UV transilluminator. For the purification purposes, the PCR product was purified by the high pure PCR Product Purification kit (Roche applied science) according to the manufacturer's recommendation.

\subsection{Cloning of P450 Cyp141}

The purified PCR product was ligated to pTZ57R/T plasmid. Then, the recombinant pTZ57R/T-cyp141 plasmid was digested with the NdeI and HindIII enzymes, which gave 1203bp release of the cytochrome P450 cyp141 fragment. In addition, a recombinant pTZ57R/T-cyp141 plasmid was 
Rabiee Faradonbeh M et al.

sent for sequence analysis (Macrogen, Korea). The released fragment was then ligated into the digested pET$26 \mathrm{~b}$ plasmid vector to construct the recombinant pET26b-cyp141 plasmid vector. The E. coli strain JM107 and $E$. coli strain BL21 (DE3) competent cells were made by the calcium chloride method (13) and used for transformation of pTZ57R/T-cyp141 and pET-26b-cyp141 vectors, respectively. The transformed bacteria were selected on LB media containing ampicillin $(50 \mathrm{mg} / \mathrm{L})$ and kanamycin (50 mg/L) antibiotics, respectively. A number of colonies were screened by colony PCR. After selecting the recombinant clones, the recombinant pET-26b-cyp141 plasmid DNA was extracted from the overnight culture and confirmed by the restriction digestion with the NdeI and HindIII enzyme analysis.

\section{Results}

\subsection{Evaluation of the Target in Terms of the Immu- nogenicity in Silico}

The in silico evaluation showed that there are a lot of hot spots regions in the cyp141 region that can be expressed as a good epitope by MHC class I and II. The results are not shown.

\subsection{Cloning and Sequencing of Cyp141}

Using the specific primers, the cyp141 gene of M. tuberculosis H37Rv was amplified, showing a band about 1200 bp on agarose gel (Figure 1). The purified PCR product (1203bp fragment) was first subcloned into pTZ57R/T vector and then for the expression of the $\mathrm{P} 450$ cyp141 protein it was cloned into the pET-26b plasmid. Following the transformation and plating of the bacterial cells on LB agar containing the above-mentioned antibiotics, some of the transformed colonies and the extracted recombinant plasmids were PCR-positive against the cyp141 gene specific primers. The recombinant plasmid (pTZ57R/Tcyp141) was confirmed by restriction digestion. Sequencing analysis (Macrogen, Korea) of the recombinant pTZ57R/T-cyp141 plasmid also confirmed that there were no amplification errors in the cloned CYP gene sequence. The sequencing result was also confirmed through comparing against databases and applying the Basic Local Alignment Search Tool.

The existence of the cyp141 gene in the pTZ57R/T-cyp141 gene and the pET26b-cyp141 gene plasmids was confirmed by digestion with NdeI and HindIII restriction enzymes. Digestion of the mentioned recombinant plasmids using the NdeI and HindIII restriction enzymes produced the two expected fragments, the 2886 bp and 1203 bp fragments for the pTZ57R/T-cyp141 gene and the $4157 \mathrm{bp}$ and $1203 \mathrm{bp}$ fragments for the pET26bcyp141 gene. Figures 2 and 3 show the recombinant plasmids after digestion.
Figure 1. Agarose Gel Electrophoresis of the Amplified CYP 141 Gene by PCR

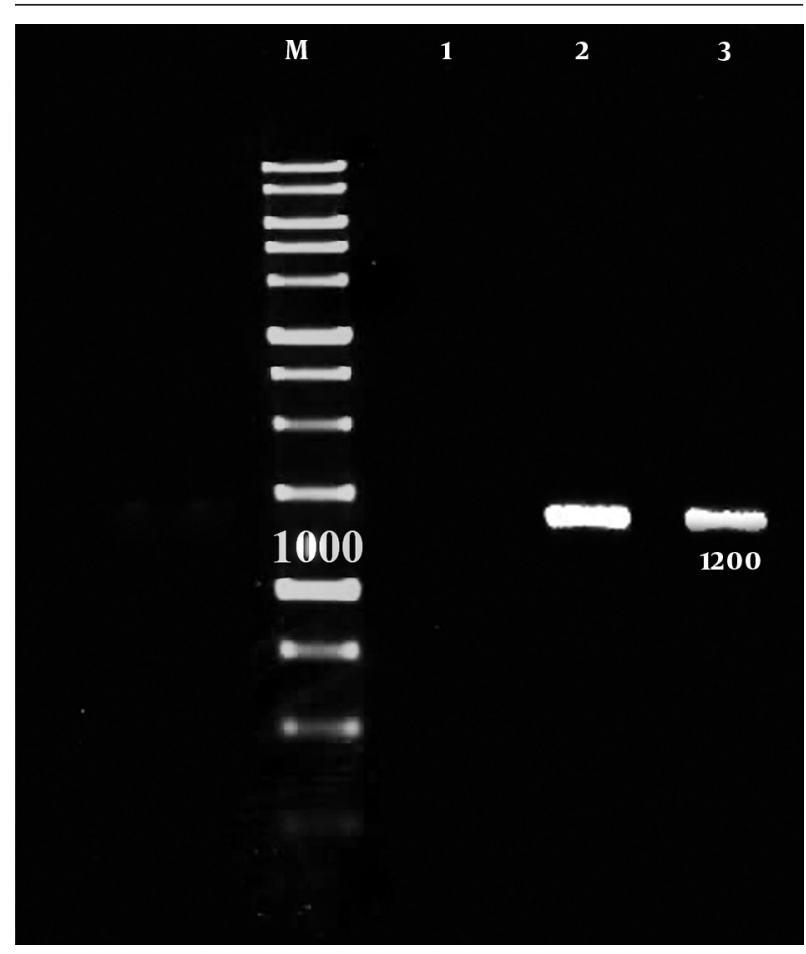

M, Molecular weight marker 1kb DNA ladder; Lane 1; negative control, Lane 2,$3 ; 1.200 \mathrm{~kb}$ PCR product.

Figure 2. Restriction Enzyme Analysis of Recombinant pTZ57R/T-CYP 141

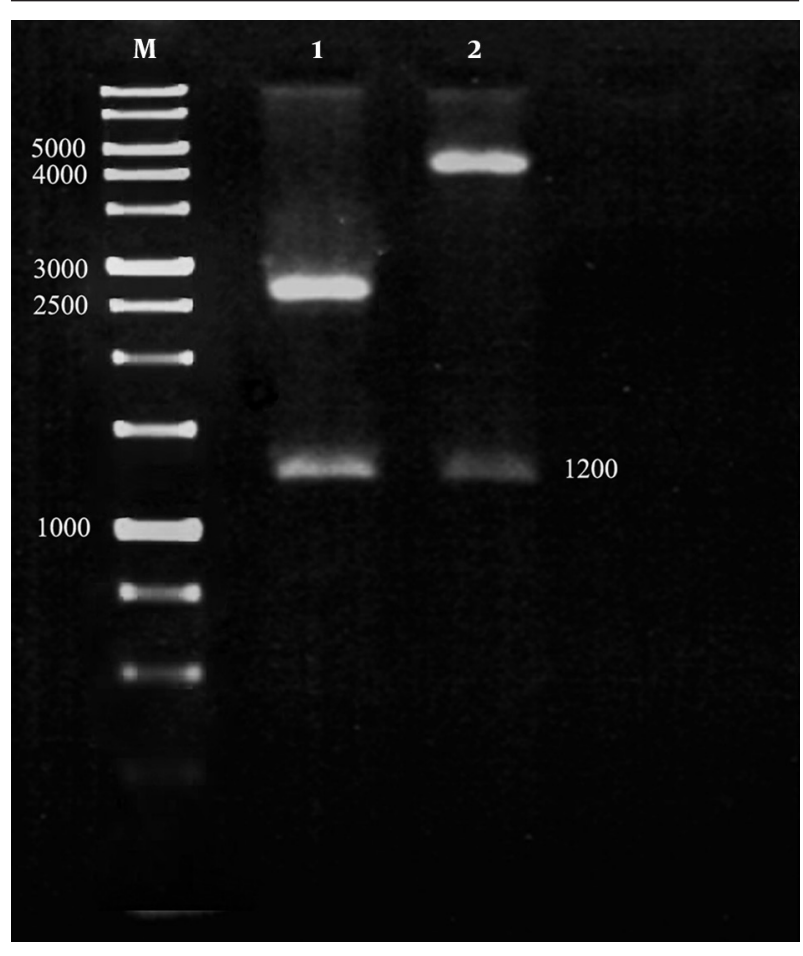

Line M, Molecular weight marker 1kb DNA ladder; Line 1 and 2, the recombinant pTZ57-R/T-CYP 141 plasmid digested by NdeI and HindIII. 
Rabiee Faradonbeh $M$ et al.

Figure 3. Restriction Enzyme Analysis of pET26b and Recombinant pET26b-CYP 141 Plasmid by NdeI and HindIII Restriction Enzymes

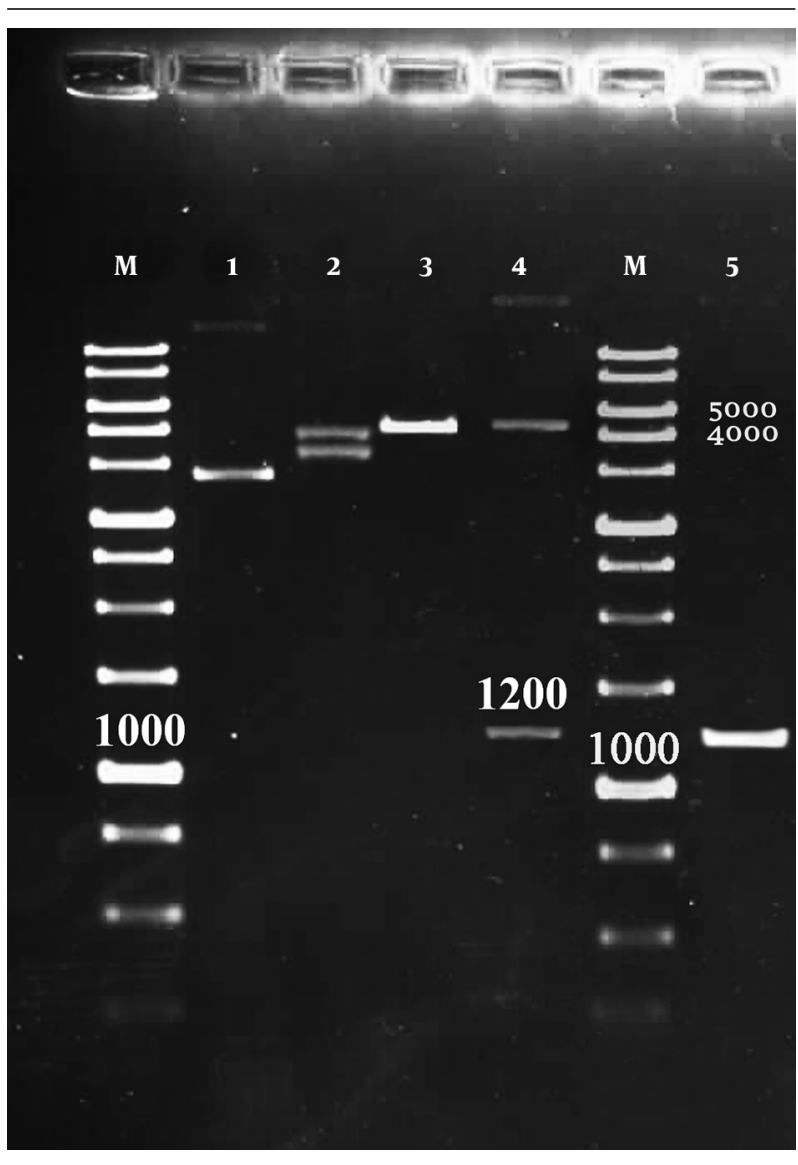

M, Molecular weight marker 1kb DNA ladder; Lane1, digested pET26b plasmid Lane 2, undigested pET26b plasmid; Lane 3, undigested pET26b-CYP 141 plasmid; Lane4, the recombinant pET26b-CYP 141plasmid digested by NdeI and HindIII (4157 bp and 1203 bp fragments); lane 5, purified PCR product.

\section{Discussion}

Identification of possible diagnostic markers and/ or vaccine candidates is a new basic science for detection and prevention of some bacterial infections and many new vaccines and diagnostic targets are presently being evaluated $(14,15)$. For example, many of bacterial genes were cloned and used effectively either as vaccines (e.g. for Brucella abortus, Bacillus anthracis, and M. tuberculosis) or as tools for the extension of antigen detection-based assays (e.g. for Streptococcus pneumonia, Legionella pneumophila, and M. tuberculosis) (1519). Tuberculosis is still one of the most important fetal infectious diseases that should be controlled. In spite of common usage of BCG vaccine to control tuberculosis, variable efficacy of BCG vaccine has led investigators to prepare another vaccine or diagnosis a candidate for this disease (20-22). Different $M$. tuberculosis genes of the immunodominant proteins have been identified and evaluated. Because these proteins can be cloned, expressed and, produced rather easily, numerous works have been focused on the expansion of different types of recombinant BCG variants and DNA vaccines (21-24). The different immunogenic components could be used for TB vaccine. The Expansion of various types of subunit vaccines, DNA vaccines and recombinant BCG variants has been evaluated in several researches $(23,25)$. The immune system can be stimulated by the recombinant BCG, recombinant protein or DNA vaccines and provides a more suitable response. In addition, some of the protein combinations alone or accompanied with other proteins and different adjuvants can lead to the defensive immune responses $(23,26,27)$. Among M. tuberculosis antigens, Ag85 complex (Ag85-A, Ag85-B, and Ag85-C), mycolyl-transferases, ESAT-6, TB10.4, and other antigens attract significant interest for the diagnostic markers and/or candidate vaccine development. Since the cytochrome P450 cyp141 protein is absent in M. bovis BCG and is possibly involved in modulation of the host immune response, it could be used as a new candidate for designing vaccines and tuberculosis diagnosis (24-26). For more evaluation of $M$. tuberculosis recombinant proteins as candidates, a stable and efficient production procedure is needed today. In addition, vaccine candidates should be prepared in the quality and quantity required for clinical applications. In this respect, a safer and more effective routine method for the production of the recombinant proteins is using the expression systems employing the hosts such as $E$. coli and plasmid vectors (23). For the purpose of the recombinant plasmid and protein production, the primers were designed for the amplification, cloning, and gene expression of the encoding frame of the cyp141 gene in a way that the amplified cyp141 fragment could be cloned in the cloning (pTZ57R/T vector) and expressing vectors (pET-26b). After the cloning of cyp141 gene in the cloning vector, the recombinant pTZ57R/T-cyp141 vector was sequenced and compared with the sequences of the cyp141 gene available in Genbank and the obtained results showed the success of cloning of the cyp141 gene into the pTZ57R/T vector. Cloning vectors such as the pTZ57R/T and the pET26 b possess the multiple cloning sites for restriction enzymes and the valence to be proliferated in the competent cells; therefore, we select those vectors for our study. Expression and purification of the cyp141 protein is ongoing and will enhance our knowledge on this recombinant protein and its potential application in the diagnosis or prevention of tuberculosis in the near future.

\section{Acknowledgements}

This study was funded by Shahrekord University of Medical Sciences with grant No. 1196 and conducted in the Department of Bacteriology, Faculty of Medicine of this University.

\section{Authors' Contributions}

Abolfazl Gholipour designed the research and col- 
laborated in writing the manuscript; Davood DarbanSarokhalil analyzed the data and wrote the paper. DNA extraction, PCR, manuscript preparation, and statistical analysis were all performed by Mohammad RabieFaradonbeh. Sample collections and coordination was performed by Amirhooshang Alvandi. In addition, Mohammad-Mehdi Feizabadi, Hasan Momtaz, and Neda Soleimani provided the extensive intellectual contribution.

\section{Funding/Support}

This study was funded by Shahrekord University of Medical Sciences with grant No. 1196 and conducted in the Department of Bacteriology, Faculty of Medicine of this University.

\section{References}

1. Gupta UD, Katoch VM, McMurray DN. Current status of TB vaccines. Vaccine. 2007;25(19):3742-51.

2. World Health Organization. WHO Report Global Tuberculosis Control: Epidemiology, Strategy, Financing. WHO; Geneva. 2012.

3. Neonakis IK, Gitti Z, Krambovitis E, Spandidos DA. Molecular diagnostic tools in mycobacteriology. J Microbiol Methods. 2008;75(1):1-11.

4. Smith R. Eradication of tuberculosis by 2050 impossible without new vaccine. BMJ. 2009;338:b1291.

5. Steingart KR, Flores LL, Dendukuri N, Schiller I, Laal S, Ramsay A, et al. Commercial serological tests for the diagnosis of active pulmonary and extrapulmonary tuberculosis: an updated systematic review and meta-analysis. PLoS Med. 2011;8(8).

6. Al-Attiyah R, Mustafa AS. Characterization of human cellular immune responses to novel Mycobacterium tuberculosis antigens encoded by genomic regions absent in Mycobacterium bovis BCG. Infect Immun. 2008;76(9):4190-8.

7. Fine PE. Variation in protection by BCG: implications of and for heterologous immunity. Lancet. 1995;346(8986):1339-45.

8. Kaufmann SH, Hussey G, Lambert PH. New vaccines for tuberculosis. Lancet. 2010;375(9731):2110-9.

9. Behr MA, Wilson MA, Gill WP, Salamon H, Schoolnik GK, Rane S, et al. Comparative genomics of BCG vaccines by whole-genome DNA microarray. Science. 1999;284(5419):1520-3.

10. McLean KJ, Dunford AJ, Neeli R, Driscoll MD, Munro AW. Structure, function and drug targeting in Mycobacterium tuberculosis cytochrome P450 systems. Arch Biochem Biophys. 2007;464(2):228-40.

11. Darban-Sarokhalil D, Fooladi AA, Bameri Z, Nasiri MJ, Feizabadi MM. Cytochrome CYP141: a new target for direct detection of Mycobacterium tuberculosis from clinical specimens. Acta Microbiol Immunol Hung. 2011;58(3):211-7.
12. Mustafa AS. Recombinant and synthetic peptides to identify Mycobacterium tuberculosis antigens and epitopes of diagnostic and vaccine relevance. Tuberculosis (Edinb). 2005;85(5-6):367-76.

13. Cohen S. Challenges influencing the future of endodontics. New technologies for endodontic education. Aust Endod J. 2001;27(3):116-8.

14. Chitlaru T, Shafferman A. Proteomic studies of Bacillus anthracis. Future Microbiol. 2009;4(8):983-98.

15. Kashino SS, Pollock N, Napolitano DR, Rodrigues V, Jr., CamposNeto A. Identification and characterization of Mycobacterium tuberculosis antigens in urine of patients with active pulmonary tuberculosis: an innovative and alternative approach of antigen discovery of useful microbial molecules. Clin Exp Immunol. 2008;153(1):56-62.

16. Sumithra TG, Chaturvedi VK, Rai AK, Chougule SS, Rajan LS, Jacob SS, et al. Progress in DNA Vaccinology against Bacterial DiseasesAn Update. Adv Anim Vet Sci. 2013;1(6):164-77.

17. Dominguez J, Gali N, Blanco S, Pedroso P, Prat C, Matas L, et al. Detection of Streptococcus pneumoniae antigen by a rapid immunochromatographic assay in urine samples. Chest. 2001;119(1):243-9.

18. Gholipour A, Moosavian M, Galehdari H, Makvandi M, Memari HR, Alvandi AH. Cloning and periplasmic expression of peptidoglycan-associated lipoprotein (PAL) protein of Legionella pneumophila in Escherichia coli. Jundishapur J Microbiol. 2010;3(1):1-9.

19. Gholipour A, Moosavian M, Makvandi M, Galehdari H, Alvandi A, Mard SA. Development of an indirect sandwich ELISA for detection of urinary antigen, using Legionella pneumophila PAL protein. World J Microbiol Biotechnol. 2014;30(5):1463-71.

20. Mori T, Leung CC. Tuberculosis in the global aging population. Infect Dis Clin North Am. 2010;24(3):751-68.

21. van Helden PD, Hoal EG. A new TB vaccine: fact or fiction? Comp Immunol Microbiol Infect Dis. 2013;36(3):287-94.

22. Jassal MS, Bishai WR. Epidemiology and challenges to the elimination of global tuberculosis. Clin Infect Dis. 2010;50 Suppl 3:S156-64.

23. Martin Montanes C, Gicquel B. New tuberculosis vaccines. Enferm Infecc Microbiol Clin. 2011;29:57-62.

24. Piubelli L, Campa M, Temporini C, Binda E, Mangione F, Amicosante $\mathrm{M}$, et al. Optimizing Escherichia coli as a protein expression platform to produce Mycobacterium tuberculosis immunogenic proteins. Microb Cell Fact. 2013;12:115.

25. Brooks JV, Frank AA, Keen MA, Bellisle JT, Orme IM. Boosting vaccine for tuberculosis. Infect Immun. 2001;69(4):2714-7.

26. Kolibab K, Yang A, Derrick SC, Waldmann TA, Perera LP, Morris SL. Highly persistent and effective prime/boost regimens against tuberculosis that use a multivalent modified vaccine virus Ankarabased tuberculosis vaccine with interleukin-15 as a molecular adjuvant. Clin Vaccine Immunol. 2010;17(5):793-801.

27. Lu J, Wang C, Zhou Z, Zhang Y, Cao T, Shi C, et al. Immunogenicity and protective efficacy against murine tuberculosis of a primeboost regimen with BCG and a DNA vaccine expressing ESAT-6 and Ag85A fusion protein. Clin Dev Immunol. 2011;2011:617892. 